\title{
THE DOUNREAY FAST REACTOR VAULT CHARACTERISATION USING THE MONTE CARLO N-PARTICLE CODE AS A TOOL TO SUPPORT DECOMMISSIONING PLANNING
}

\author{
Vittoria Baldioli $^{1}$, Richard Gunn ${ }^{1}$, Sanders MacDonald ${ }^{1}$ and Aly Mackay ${ }^{1}$ \\ ${ }^{1}$ Dounreay Site Restoration Ltd \\ Dounreay, Thurso, KW14 7TZ
}

Vittoria.baldioli@dounreay.com, Richard.gunn@dounreay.com, Sanders.macDonald@dounreay.com,Aly.mackay@dounreay.com

\begin{abstract}
A combined empirical and theoretical characterisation approach was taken in order to investigate the activity distribution and inventory in the Dounreay Fast Reactor (DFR) vault, so as to aid with the decommissioning and waste management strategy of the cooling circuits located within the shielded vault. The DFR vault is a no-man access, shielded void, which surrounds the reactor core and the graphite shield.

Empirical data was collected through various Non-Destructive Assay methods, utilising all available accesses into the reactor vault. This collection of information was then used to make a first assumption about the activity distribution within the vault; this was then included into a MCNP model of the vault as the source terms.

The model was run and a dose rate map of the vault obtained, and compared to the empirical dose rate data previously gathered in the accessible penetrations in the vault; the model was altered until a satisfactory agreement was obtained between theoretical and measured data. Once this was obtained then, the model was used to provide an estimate of the activity inventory within the vault, and specifically within the various reactor components.
\end{abstract}

KEYWORDS: MCNP, Decommissioning, Fast Reactor

\section{INTRODUCTION}

The Dounreay Fast Reactor, known as DFR, was an experimental fast reactor which became critical for the first time in 1959 and achieved its design power output of $60 \mathrm{MW}$ thermal in 1963. On completion of the experimental programme, the reactor was finally shut down in 1977, entering periods of care and maintenance and decommissioning. Due to its nature, the decommissioning of the Dounreay Fast Reactor presents one of the most significant and hazardous challenges in the UK nuclear industry today, and it is of paramount importance to the project that radiation levels and activity distribution within the reactor vault are well understood.

To optimise and aid the decommissioning and waste management strategy of the cooling circuits located within the shielded reactor vault, a characterisation campaign was launched. This involved various nondestructive assay methods, as well as the construction of a model of the reactor vault using the Monte Carlo N-Particle code, in order to obtain an understanding of the activity distribution within the vault. The data collected through methods of gamma imaging and gamma spectroscopy were used to derive the initial source term constructed in the model; this was then run with the inclusion of tallies, to which dose 
rate modifiers were applied. The tally points were placed in the model at positions within the vault at which dose rates had been measured; in order for the data to be representative of the entire reactor vault, dose rate data were collected at every accessible penetration into the vault. The dose rates obtained from the model run were then compared to the measured dose rates, and the model was subsequently iteratively altered and re-run until a close match between the empirical and computed dose rates was achieved, indicating the correct activity distribution within the vault had been modelled. Moreover, a close match also provides validation for the model.

Furthermore, from the comparison and manipulation of the measured and modelled dose rates, the number of starting photons was calculated for the reactor vault; this was then used to provide an estimate of the total $137 \mathrm{Cs}$ activity inventory within the vault. From the spatial representation of the activity distribution obtained from the model then, the 137Cs activity inventory of various items within the reactor vault could also be estimated.

The model created in this project thus serves as a collection and interpretation of the data obtained through the various characterisation campaigns performed, by providing a spatial representation of the activity distribution within the reactor vault, as well as an inventory estimate. This information and model can now be used to support decision making for the reactor decommissioning project, to minimise operator dose uptake during decommissioning operations, as well as to enable assessments as to whether the operations can be performed manually or remotely. The model may also be used as a decommissioning tool and modified in the future to determine the effect of shielding and equipment removal scenarios on the radiation dose rates within/outside the vault.

\section{METHODOLOGY}

The characterisation of the DFR vault discussed in this work can be divided in two broad stages: empirical data gathering, and modelling work; the two methodologies were then combined.

\subsection{Empirical Data Gathering through Non Destructive Assay Methods}

A series of campaigns were carried out in order to gather empirical data in the reactor vault, employing various Non-Destructive Assay (NDA) methods, depending on suitability and accessibility into the vaults at various locations.

Firstly, a gamma spectroscopy campaign was performed, by deploying a CdZnTe detector, which was suitable for this application as, due to its compact dimensions, it could be deployed in several available penetrations around the vault. This included umbrella heaters penetrations and redundant expansion tanks penetrations. This was deployed at various depths in each penetration, in conjunction with a custom-built collimator, and rotated by $360^{\circ}$ at each position, in order to obtain radial directional information on the activity recorded.

An STHF-R ${ }^{\mathrm{TM}}$ ultra-high flux gamma probe [1] was also deployed in the same penetrations and depthsagain, thanks to its compact size- and a dose rate reading recorded for each location. A combination of the two readings provided good qualitative and quantitative information on the activity and dose rates across the vault. A total of 130 dose rate measurements were acquired across the vault.

In addition to the above, a maintenance access point was available for use, and due to its larger dimensions, the RadScan gamma imager [2] was able to be deployed at this point. Moreover, two expansion tank probe pockets were accessible, and the N-Visage gamma imager [3] - more compact than the RadScan- was deployed in these two locations around the vault. These three measurements enabled to obtain both optical images - which were of interest in themselves, since physical man access is prohibited in the vault- and a radiometric overlay of the distribution of activity over the various components within the vault. This represented a useful description of the activity distribution and, in conjunction with the 
measurements acquired as discussed above, enabled a first approximation of the source term within the MCNP model.

\subsection{MCNP Modelling of the Reactor Vault}

An MCNP model of the reactor vault was constructed using MNCP6.2 [4]. The elements included in the model were the ones that were considered to be relevant to the assessment; i.e. the model aimed at being sufficiently detailed for radiation transport purposes but compromising on a reasonable computational time. The core and graphite, expansion tanks, bypass vessels, primary heat exchangers, the ring main and the redundant nitrogen line were modelled in the vault. Figure 1 shows the model in its entirety (left) and with the outside walls emitted (right) for clarity, as represented in MCNP Visual Editor [5].


Figure 1. DFR vault geometry model, as displayed in VisEd.

The source terms defining the source locations, relative intensities, energies and physical geometry, were included in the code based on the information gathered through the empirical measurement campaigns discussed above. The sources were described in the code as being $100 \%{ }^{137} \mathrm{Cs}$, as this had been previously observed to be a good approximation of the case, and the main contributor to dose rates.

Tallies of the F5 type and associated flux to dose rate conversion factors were included in the model, in positions that reflected the exact positions at which dose rate measurements were taken with the STHF High flux probe. This would allow a meaningful comparison between the empirical values and the ones calculated with MCNP. A good agreement, following iterative runs altering the source term, would provide a validation of the model. The tallies were located in the model as displayed in figure 2 . 


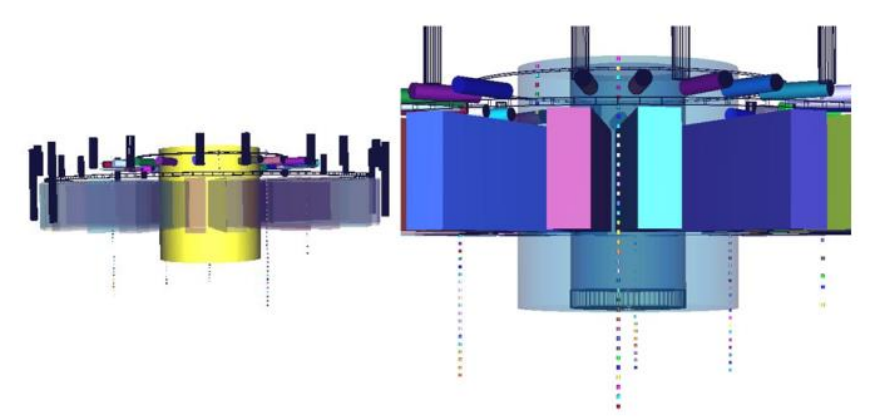

Figure 2. Visual representation of detector positions at various penetrations around the vault, as plotted in VisEd.

\section{RESULTS AND DISCUSSION}

\subsection{Empirical Data Gathered}

The main sources of activity, identified through the gamma spectroscopy and gamma imaging measurements, were the expansion tanks, heat exchangers, bypass vessels, ring main, and the nitrogen line. The gamma dose rate readings taken were then seen to be consistent with these findings.

\subsection{MCNP Modelling Results}

The modelling work can be divided into two main tasks: model set-up and validation, through the comparison with empirical dose rate data; model runs to calculate the activity distribution and inventory within the reactor vault.

\subsubsection{Model validation - empirical and theoretical data}

The MCNP model of the reactor vault was, once set up as described above, run with an initial hypothesis of the distribution of the activity within the vault, based on the empirical data acquired. At this stage, the MNCP results were obtained in terms of average dose rate per starting photon; both these and the empirically measured dose rates were thus normalized to 1 in order to allow for direct comparison between the theoretical and the measured dose rates at each location investigated. The comparisons were then studied and, based on good or poor correlations at the various positions, the model was re-run by altering the source term accordingly -i.e. altering the amount of activity associated with items at different locations.

A satisfactory correlation between empirical and theoretical dose rates was eventually obtained through this process. Generally, the modelled and measured values followed the same trend and, comparing the normalized values, percentage differences between 0-30\% were obtained generally, which was considered to be a good and satisfactory agreement given the simplifications of the model. Some outliers were observed, but some were expected since, as mentioned, the model is a simplified approximation of the real situation, and certain contamination hotspots might be present in areas that were not empirically surveyed, and therefore not included as a source in the model.

This part of the work provided a description of the distribution of activity in terms of fractions of activity in the various components in the vault, as well as confirmation of the model validity, given the good agreement obtained between measured and modelled data. 


\subsubsection{Activity distribution and inventory}

From the model as set up and validated as discussed above, it was desired to calculate an activity inventory for the vault for ${ }^{137} \mathrm{Cs}$, and for individual reactor components.

To this end, the number of starting photons was calculated for each position as displayed in equation (1) below, and an average of all positions was then taken.

$$
\text { Total starting photons }=\frac{\text { Measured dose rate }(\mathrm{mSv} / \mathrm{h})}{\text { MCNP output }(\mathrm{mSv} / \mathrm{h} / \text { starting photon })},
$$

This number of starting photons was then converted to a value of total activity in each cell, with equation (2)

$$
\text { Total Activity }(B q)=\text { Total starting photons } \times \text { emission probability }
$$

Where the emission probability or branching fraction for the $661.67 \mathrm{keV}$ emission for ${ }^{137} \mathrm{Cs}$, which is assumed in this situation to comprise $100 \%$ of the activity, is 0.8499 . [6]

The inventory in each major component in the reactor vault was then calculated, using the total activity as calculated with equation 2 , and the fraction of activity in each item as established during the model validation process.

\section{CONCLUSIONS}

An investigation into the distribution of activity within the shielded vault of the Dounreay Fast Reactor was carried out by combining empirical and theoretical methods.

Firstly, data was gathered through various means of non-destructive assay, including gamma spectroscopy and gamma imaging, and dose rate surveys were performed through all accessible penetrations in the vault with a gamma dose rate probe.

The data acquired during these measurement campaigns were collated and used to inform the creation of a source term in a Monte Carlo N-Particle model of the reactor vault. This model was then run with the inclusion of F5 tallies with dose rate modifiers; the model was run multiple times by altering the activity distribution in each vault component, which included expansion tanks, heat exchangers, bypass vessels, ring main, and the nitrogen line, until a good correlation was obtained between empirical dose rates, and modelled dose rates. The modelled and measured values were seen to follow the same trend in each case and, when both sets of data were normalised and compared directly, percentage differences between 0 $30 \%$ were obtained. This was considered to be a good and satisfactory agreement given the necessary simplifications of the model, in place to compromise between accuracy of model and computational time. This phase offered not only a validation of the model, but also a description of the activity distribution amongst the vault components. The total activity due to ${ }^{137} \mathrm{Cs}$ within the vault, as well as the inventory for each component were then calculated.

As a result of this work, the DFR decommissioning project team now have an estimate of the activity inventory within the vault, as well as a collection of data and a MCNP model that can be modified and corrected in the future as more data becomes available e.g. if more measurement campaigns are carried out. The model can be used for dose budgeting and to assess the necessity for manual or remote operations. Finally, the model can be used as a decommissioning tool, modified when necessary, and used to determine dose rates within or outside the vault through the effects of shielding and during component removal scenarios. 


\section{REFERENCES}

1. Mirion Technologies, Canberra UK (ltd), STHF-R ${ }^{\mathrm{TM}}$ Ultra High Flux Gamma Probe (2017).

2. BNFL Instruments, RadScan $800^{\circledR} 4$ pi Gamma Imager (2002).

3. CREATEC, N-Visage ${ }^{\mathrm{TM}}$ Scanner.

4. C.J. Werner, et al., "MCNP6.2 Release Notes", Los Alamos National Laboratory, report LA-UR-1820808 (2018).

5. A. L. Schwarz, R. A. Schwarz, and A. R. Schwarz, "MCNPX/6® Visual Editor Computer Code Manual" (January 2018).

6. M.-M. Bé et al., "Monographie BIPM-5",Table of Radionuclide, Vol. 3, pp. 91-96, BUREAU INTERNATIONAL DES POIDS ET MESURES, Pavillon de Breteuil, F-92310 SĖVRES (2006). 